\title{
Bending dynamics of viscoelastic photopolymer nanowires
}

Cite as: Appl. Phys. Lett. 117, 013701 (2020); https://doi.org/10.1063/5.0014662

Submitted: 20 May 2020. Accepted: 22 June 2020 . Published Online: 06 July 2020

Jana Kubacková, Gergely T. Iványi, Veronika Kažiková, Alena Strejčková, Andrej Hovan, Gabriel Žoldák (D), Gaszton Vizsnyiczai (D), Lóránd Kelemen (D), Zoltán Tomori (D), and Gregor Bánó (iD)

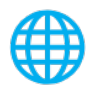

\section{Lock-in Amplifiers up to $600 \mathrm{MHz}$}
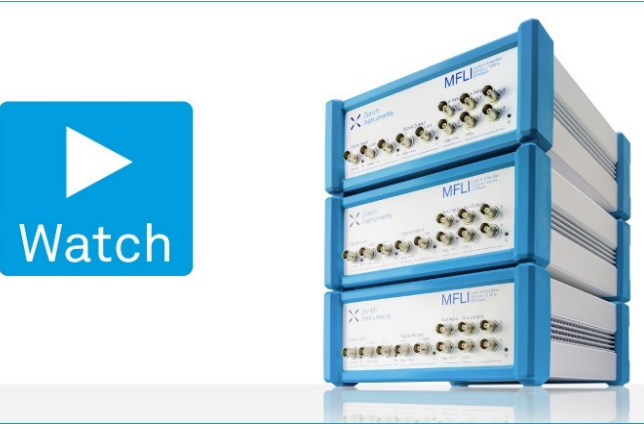


\title{
Bending dynamics of viscoelastic photopolymer nanowires
}

\author{
Cite as: Appl. Phys. Lett. 117, 013701 (2020); doi: 10.1063/5.0014662 \\ Submitted: 20 May $2020 \cdot$ Accepted: 22 June 2020 . \\ Published Online: 6 July 2020

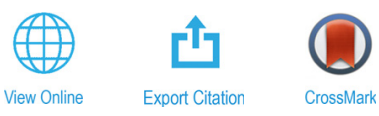

Jana Kubacková, ${ }^{7}$ Gergely T. Iványi, ${ }^{2}$ Veronika Kažiková, ${ }^{7}$ Alena Strejčková, ${ }^{3}$ Andrej Hovan, ${ }^{4}$ Gabriel Žoldák, Gaszton Vizsnyiczai, ${ }^{2}$ (D) Lóránd Kelemen, ${ }^{2}$ (D) Zoltán Tomori, ${ }^{1}$ (D) and Gregor Bánó ${ }^{4,5, a)}$ (iD

\author{
AFFILIATIONS \\ ${ }^{7}$ Department of Biophysics, Institute of Experimental Physics, Slovak Academy of Sciences, Watsonova 47, 040 01 Košice, \\ Slovak Republic \\ ${ }^{2}$ Institute of Biophysics, Biological Research Centre, Temesvári krt. 62, Szeged, Hungary \\ ${ }^{3}$ Department of Chemistry, Biochemistry and Biophysics, Institute of Biophysics, University of Veterinary Medicine and Pharmacy, \\ Komenského 73, 04181 Košice, Slovak Republic \\ ${ }^{4}$ Department of Biophysics, Faculty of Science, P. J. Šafárik University, Jesenná 5, 04154 Košice, Slovak Republic \\ ${ }^{5}$ Center for Interdisciplinary Biosciences, Technology and Innovation Park, P. J. Šafárik University, Jesenná 5, 04154 Košice, \\ Slovak Republic
}

${ }^{a)}$ Author to whom correspondence should be addressed: gregor.bano@upjs.sk

\begin{abstract}
In this work, we demonstrate that the mechanical dynamics of polymer nanowires prepared by two-photon polymerization direct laser writing lithography is strongly influenced by their viscoelastic characteristics. Bending recovery measurements were carried out on cantilevered nanowires deflected by optical tweezers in a liquid environment. The assumption of purely elastic cantilever response (as defined by Young's modulus of the polymer material) fails to explain the observed overdamped oscillatory motion. A mechanical model is proposed to account for the nanowire viscoelastic behavior. The experimental data indicate that the origin of the nanowire viscous component is twofold. Both the partially cross-linked polymer structure and the solvent penetrating the polymer network contribute to frictional forces inside the nanowire. The present results provide guidance for the future design of nanosized polymer devices operated in a dynamic regime.
\end{abstract}

Published under license by AIP Publishing. https://doi.org/10.1063/5.0014662

Owing to the high flexibility and the nanoscale spatial resolution of two-photon polymerization direct laser writing (TPP-DLW) lithography, ${ }^{1-6}$ the application field of nano- and microstructures fabricated by this technique is growing fast. Recent developments cover the areas of photonic devices, ${ }^{7-9}$ nano-micro-mechanics, ${ }^{10-12}$ and biomedical applications. ${ }^{13-15}$ To exploit the full potential of TPP-DLW lithography, the mechanical properties of the prepared structures must be well characterized, which were the subject of several previous works. ${ }^{16}$ Special attention was paid to the scaling of photopolymer elastic moduli (Young's modulus and/or the shear modulus) when moving from the bulk material to micro- and nanoscale dimensions. Depending on the experimental conditions, which include the type of the used photoresist, the structure dimensions, the polymerization parameters (laser power, writing speed, and post-curing settings), and the surrounding environment (solution or air), the elastic modulus difference between the bulk and microscopic objects ranged from a factor of few times, ${ }^{17}$ up to three orders of magnitude. ${ }^{18}$ Moreover, opposing scaling of the elastic moduli (increase or decrease) was reported toward smaller object features at different experimental conditions. ${ }^{19-22}$

The higher elastic moduli observed in nanoscale objects, compared to bulk material, were explained by the enhanced alignment of the polymer network. ${ }^{19,20}$ By contrast, the chemical and physical phenomena identified behind the opposite effect (i.e., lowered material stiffness of nanoscale structures) are the limited degree of polymer cross-linking and the enhanced solvent permeation into the polymer network. Indeed, Raman and CARS (coherent anti-Stokes Raman scattering) spectroscopy revealed a significant portion of uncured resin in the structures prepared by TPP-DLW at laser powers near the polymerization threshold [Fig. 1(a)]. ${ }^{21-24}$ The degree of conversion increased toward higher laser powers and/or lower writing speeds. In this way, the structure stiffness could be enhanced, usually at the expense of the fabrication spatial resolution..$^{20-22,24,25}$ Near the polymer surface, the remnant monomers, oligomers, and incompletely polymerized chains are removed from the material by the developer, 
a) near-threshold irradiation

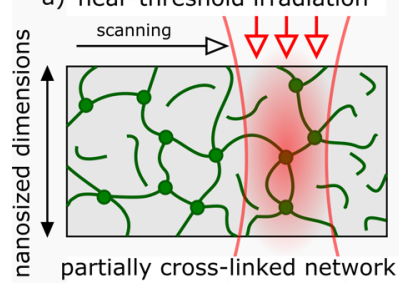

b) liquid environment

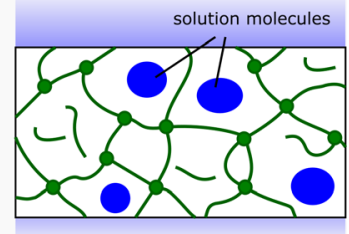

swollen polymer network

FIG. 1. Schematic view of the polymer network inside the nanowires prepared by TPP-DLW. (a) The partially cross-linked polymer network during the polymerization process. (b) Solution molecules penetrate the nanowire structure when placed in a liquid environment.

which is mostly important in the case of nanosized objects with a large surface-to-volume ratio. The structure-loosened polymer state makes the nanostructures permeable to solvent molecules [Fig. 1(b)], thus affecting the stiffness, shrinkage, and swelling characteristics of the material. ${ }^{26,27}$ The question arises whether or not the viscous character of the uncured resin and the penetrated solvent affects the mechanical properties of the nanostructures prepared by TPP-DLW. Optical tweezers, ${ }^{18,21,28}$ nanoindentation equipment, ${ }^{24,25}$ atomic force microscopes, ${ }^{17,19,29}$ and, more recently, micro-electro-mechanical systems (MEMS)-based tensile test$\mathrm{ers}^{20}$ and nanorobotic systems ${ }^{22}$ were utilized to follow the photopolymer response to the applied mechanical stress. With a few exceptions, all the results used for elastic modulus evaluation were obtained at quasisteady conditions. In this work, the bending characteristics of photopolymer nanowires are studied in a dynamic regime. Based on the recovery motion of cantilevered nanowires (bent with optical tweezers), the viscoelastic material characteristics are explored and the related aspects of photopolymer nanomechanics are uncovered.

$18 \mu \mathrm{m}$ long polymer nanowires, anchored to vertical supports at a height of $8 \mu \mathrm{m}$ and equipped with $5 \mu \mathrm{m}$ diameter trapping spheres [Figs. 2(a) and 2(b)], were used for bending dynamics measurements. The cantilevered nanowires were fabricated using TPP of Ormocomp, the commercially available biocompatible inorganic-organic hybrid polymer. ${ }^{15,30-32}$ The $785 \mathrm{~nm}, 100 \mathrm{MHz}$ repetition rate, and $100 \mathrm{fs}$ pulse length polymerization laser was focused into the photoresist by a $40 \times$ oil immersion objective (NA 1.3). The nanowire was drawn as a single line with a scan speed of $50 \mu \mathrm{m} / \mathrm{s}$. The laser power was set to a nearthreshold value of $3.5 \mathrm{~mW}$ in the sample. In order to stabilize the nanowire position for the development, washing, and drying processes, an identical cantilever was polymerized to the opposite side of the sphere [Fig. 1(c)]. The auxiliary cantilever was cut and removed from the structure before the measurements. The structures were washed in OrmoDev developer three times for $15 \mathrm{~min}$. While immersed in the third developer, the coverslip was irradiated with a microscope mercury lamp (HBO50) to promote post-polymerization. Finally, the structures were washed in water and air-dried. The width and the height of the dried nanowires were ca. $150 \mathrm{~nm}$ and $400 \mathrm{~nm}$, respectively.

The cantilevers immersed in aqueous solutions were deflected horizontally by optical forces exerted on the sphere [Fig. 2(a)], keeping the deflection angle below $170 \mathrm{mrad}$. After switching the trapping laser off, the cantilevers moved back to the initial equilibrium in an overdamped oscillatory regime. The analysis of the recovery motion was used to determine the cantilever's viscoelastic characteristics, without the need for the knowledge of the exerted optical force. The sphere

a)
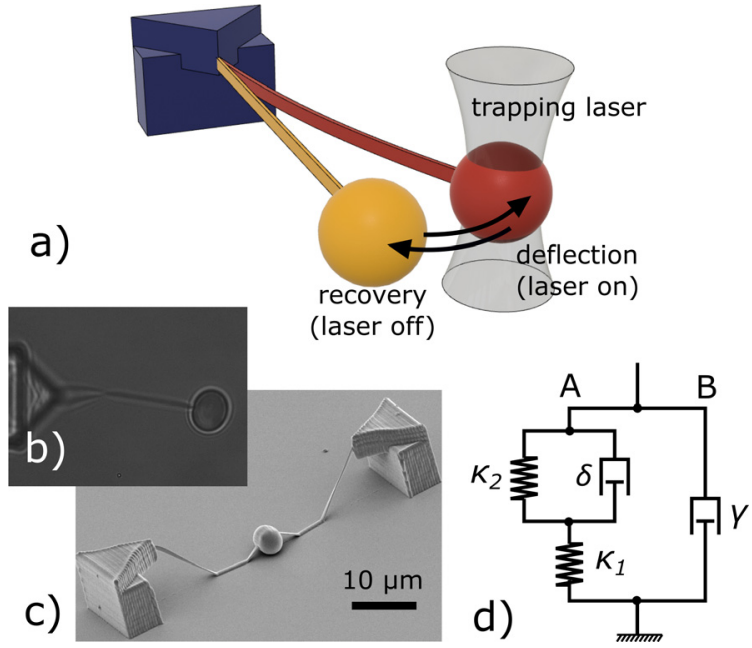

FIG. 2. (a) The bending recovery experiment. (b) Bright-field picture of the cantilevered nanowire in water. (c) SEM picture of the dried cantilever. The right side of the structure was cut and removed before the experiments. (d) The mechanical model for the sphere motion along the recovery trajectory. The A and B arms represent the nanowire and the hydrodynamic damping by the surrounding medium, respectively.

position was followed by video tracking, using a CMOS camera operated at $500 \mathrm{fps}$. The deflection/recovery procedure was repeated twelve times, and the average distance traveled by the sphere along the recovery trajectory was evaluated. The viscosity of the surrounding medium was tuned by using aqueous glucose solutions of different concentrations. ${ }^{33}$

The normalized time courses of the sphere displacement are shown in Fig. 3(a). The recovery is driven by the nanowire elastic forces, acting toward the equilibrium, and the counter-acting dissipative forces. As expected, the sphere motion slows down when the medium viscosity is increased at higher glucose concentrations. It is important to note that the time dependence of the recovery curves exhibits a bi-exponential decay. This observation contradicts the assumption of purely elastic nanowires. The discrepancy is resolved by taking the viscoelastic nanowire characteristics into account. The mechanical model proposed in this work for the sphere connected to the nanowire is shown in Fig. 2(d). The model consists of spring and dashpot elements. The left arm (assigned as A) represents the viscoelastic properties of the nanowire. The proposed scheme resembles the standard linear solid model of viscoelastic materials. In our case, however, the two spring constants, $\kappa_{1}$ and $\kappa_{2}$, and the viscoelastic damping coefficient $\delta$ are effective parameters assigned to the cantilevered nanowire as a whole. The B arm stands for the viscous forces of the surrounding medium, with $\gamma$ denoting the hydrodynamic resistance. Neglecting the cantilever thickness compared to its length and the sphere diameter, the model system of Fig. 2(d) is described by the sphere equation of motion,

$$
m \frac{\mathrm{d}^{2} x}{\mathrm{~d} t^{2}}=F_{\mathrm{A}}+F_{\mathrm{B}},
$$

where $x$ is the position of the sphere on the deflection trajectory and $m$ is the sphere mass. $F_{\mathrm{A}}$ and $F_{\mathrm{B}}$ are the viscoelastic and viscous forces exerted by the cantilever and the solution, respectively. In the case of 

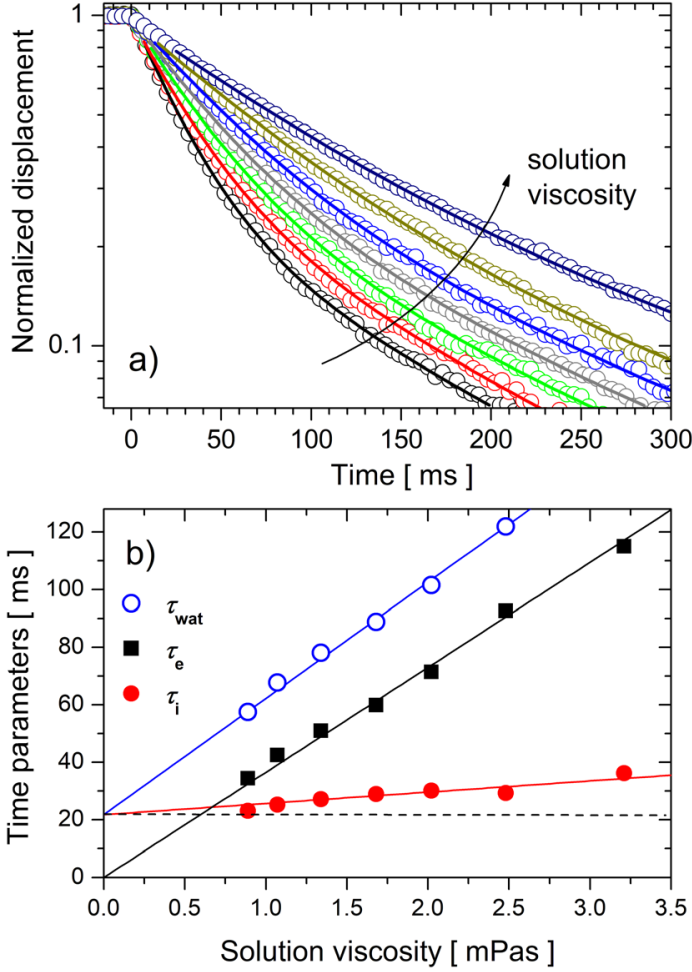

FIG. 3. (a) The displacement recovery curves measured in glucose solutions of 0 , $100,150,200,250,300$, and $350 \mathrm{mg} / \mathrm{ml}$ concentration. The solid lines represent biexponential fits. All the curves were normalized to unity. (b) The weighted average time $\tau_{\text {wat }}$ (open blue circles) and its two terms, $\tau_{\mathrm{e}}$ (solid black squares) and $\tau_{\mathrm{i}}$ (solid red circles) plotted as a function of the solution viscosity.

micrometer-sized objects, the inertial forces can be neglected, ${ }^{34,35}$ and Eq. (1) is solved analytically. The calculation details are given in the supplementary material. In good agreement with the experimental observations, the theoretical time dependence of the nanowire deflection during the overdamped oscillation has a bi-exponential form,

$$
x(t)=A_{1} e^{-\frac{t}{\tau_{1}}}+A_{2} e^{-\frac{t}{\tau_{2}}} .
$$

The decay times $\tau_{1}$ and $\tau_{2}$, and the amplitudes $A_{1}$ and $A_{2}$ are determined by fitting the experimental recovery curves with Eq. (2). The relations between the four fitting parameters $\left(\tau_{1}, \tau_{2}, A_{1}\right.$, and $\left.A_{2}\right)$, the cantilever characteristics $\left(\kappa_{1}, \kappa_{2}\right.$, and $\left.\delta\right)$, and the hydrodynamic resistance $(\gamma)$ have a relatively complex form. The data analysis simplifies significantly when the weighted average time $\tau_{\text {wat }}$ is calculated,

$$
\tau_{\text {wat }}=\frac{A_{1} \tau_{1}+A_{2} \tau_{2}}{A_{1}+A_{2}}
$$

As shown in the supplementary material, the weighted average time can be divided into two terms, $\tau_{\mathrm{i}}$ and $\tau_{\mathrm{e}}$, which separate the internal and external friction in the system,

$$
\begin{gathered}
\tau_{\text {wat }}=\tau_{\mathrm{i}}+\tau_{\mathrm{e}}, \\
\tau_{\mathrm{i}}=\left(\frac{1}{\kappa_{2}}-\frac{1}{\kappa_{1}+\kappa_{2}}\right) \delta,
\end{gathered}
$$

$$
\tau_{\mathrm{e}}=\left(\frac{1}{\kappa_{1}}+\frac{1}{\kappa_{2}}\right) \gamma
$$

The value of $\tau_{\mathrm{i}}$ is proportional to the internal damping coefficient $\delta$ and carries information about the nanowire viscous component. By contrast, $\tau_{\mathrm{e}}$ is a linear function of the hydrodynamic resistance $\gamma$ and characterizes the behavior of a hypothetical, purely elastic nanowire. To gain relevant information about the nanowire viscoelasticity, the values of $\tau_{\mathrm{i}}$ and $\tau_{\mathrm{e}}$ must be determined separately. Here, we take advantage of the fact that both $\tau_{\mathrm{i}}$ and $\tau_{\mathrm{e}}$ can be calculated directly, using the four fitting parameters

$$
\begin{gathered}
\tau_{\mathrm{i}}=\frac{A_{1} A_{2}\left(\tau_{2}-\tau_{1}\right)^{2}}{\left(A_{1}+A_{2}\right)\left(A_{1} \tau_{2}+A_{2} \tau_{1}\right)}, \\
\tau_{\mathrm{e}}=\frac{\left(A_{1}+A_{2}\right) \tau_{1} \tau_{2}}{A_{1} \tau_{2}+A_{2} \tau_{1}} .
\end{gathered}
$$

For spherical particles, the hydrodynamic resistance $\gamma$ depends on the bead radius $a$ and the solution viscosity $\eta, \gamma=6 \pi \eta a$. In the case of the studied cantilever systems, corrections are to be made for the finite distance of the sphere from the chamber wall [the resistance is estimated to increase by $\sim 20 \%$ (Ref. 36)] and also for the viscous drag exerted on the cantilever beam. Taking all these corrections into account, the hydrodynamic resistance remains proportional to the medium viscosity. The proportionality factor between $\gamma$ and $\eta$ is, however, difficult to express analytically.

The normalized time courses of the sphere displacement [Fig. 3(a)] were fitted with bi-exponential decays. The fitting parameters $\left(\tau_{1}, \tau_{2}, A_{1}\right.$, and $\left.A_{2}\right)$ were used to evaluate the weighted average time $\tau_{\text {wat }}$ and its two terms, $\tau_{\mathrm{i}}$ and $\tau_{\mathrm{e}}$. The results are plotted in Fig. 3(b) as a function of the surrounding solution viscosity. In agreement with the proposed model, $\tau_{\mathrm{e}}$ is a linear function of the viscosity and the extrapolation of the $\tau_{\mathrm{e}}$ values toward zero viscosity passes through the $(0,0)$ point. This result shows that the effective cantilever stiffness [defined as $1 / \kappa_{\text {eff }}=1 / \kappa_{1}+1 / \kappa_{2}$, see Eq. (4c)] is a constant. The nanowire stiffness and, in general, the elastic material response are determined by the cross-linked polymer network. Obviously, the solution viscosity has a negligible effect on this network. It is reasonable to assume that the two elastic terms, $\kappa_{1}$ and $\kappa_{2}$ separately, are also constants. Taking Eq. (4b) into account, it follows that the $\tau_{\mathrm{i}}$ values, plotted in Fig. 3(b) with solid red circles, reflect the changes in the nanowire damping coefficient $\delta$. Based on the experimental data, we conclude that there are two effects contributing to the nanowire internal damping. The first contribution is independent of the solution viscosity (indicated by the dashed line in Fig. 3) and, most probably, corresponds to the uncured part of the photopolymer. The second contribution, which is proportional to the solution viscosity, is assigned to the solution molecules penetrating the loosened polymer structure. It is concluded that both the limited degree of polymer cross-linking and the solvent permeation into the polymer network affect the viscoelastic material characteristics of photopolymerized nanowires. The relative importance of the two contributions may, however, be different for each particular case, depending on the fabrication conditions.

The mechanical properties of nano-micro-oscillators fabricated by TPP-DLW have been investigated for almost twenty years now. The present work extends the nanowire mechanics toward the dynamic regime. The viscoelastic nanowire behavior, identified and described here, must be taken into account when designing 
nanomechanical components used in dynamic stress conditions. A deeper understanding of the material properties opens new possibilities for future applications of nanostructures prepared by TPP-DLW. Microrheological measurements can be mentioned as an example. The present cantilevered nanowires can be used as microscopic viscometers in a straightforward way.

See the supplementary material for the computational details of the mechanical model.

This work was supported by the Slovak Research and Development Agency (Grant Nos. APVV-15-0665 and APVV-18-0285), the Slovak Ministry of Education (Grant KEGA No. 012 UVLF-4/2018), the joint project of Slovak and Hungarian Academies of Sciences (No. NKM-88/ 2019), and the GINOP-2.3.2-15-2016-00001 and the GINOP-2.3.3-152016-00040 programs. This project also received funding from the European Union's Horizon 2020 research and innovation program under Grant Agreement No. 654148 Laserlab-Europe. We gratefully acknowledge the support of NVIDIA Corporation with the donation of the Titan Xp GPU used for this research.

\section{DATA AVAILABILITY}

The data that support the findings of this study are available within the article (and its supplementary material).

\section{REFERENCES}

${ }^{1}$ M. Malinauskas, M. Farsari, A. Piskarskas, and S. Juodkazis, "Ultrafast laser nanostructuring of photopolymers: A decade of advances," Phys. Rep. 533, 1-31 (2013).

${ }^{2}$ C. N. LaFratta, J. T. Fourkas, T. Baldacchini, and R. A. Farrer, "Multiphoton fabrication," Angew. Chem.-Int. Ed. 46, 6238-6258 (2007).

${ }^{3}$ L. J. Li, R. R. Gattass, E. Gershgoren, H. Hwang, and J. T. Fourkas, “Achieving $\lambda / 20$ resolution by one-color initiation and deactivation of polymerization," Science 324, 910-913 (2009).

${ }^{4}$ D. Grafe, S. L. Walden, J. Blinco, M. Wegener, E. Blasco, and C. BarnerKowollik, "It's in the fine print: Erasable three-dimensional laser-printed micro- and nanostructures," Angew. Chem.-Int. Ed. 59, 6330-6340 (2020).

${ }^{5}$ R. Wollhofen, B. Buchegger, C. Eder, J. Jacak, J. Kreutzer, and T. A. Klar, "Functional photoresists for sub-diffraction stimulated emission depletion lithography," Opt. Mater. Express 7, 2538-2559 (2017).

${ }^{6} \mathrm{~J}$. Fischer and M. Wegener, "Three-dimensional optical laser lithography beyond the diffraction limit," Laser Photonics Rev. 7, 22-44 (2013).

${ }^{7}$ G. Zyla, A. Kovalev, M. Grafen, E. R. Gurevich, C. Esen, A. Ostendorf, and S. Gorb, "Generation of bioinspired structural colors via two-photon polymerization," Sci. Rep. 7, 17622 (2017).

${ }^{8}$ A. Camposeo, L. Persano, M. Farsari, and D. Pisignano, "Additive manufacturing: Applications and directions in photonics and optoelectronics," Adv. Opt. Mater. 7, 1800419 (2019).

${ }^{9}$ T. Gissibl, S. Thiele, A. Herkommer, and H. Giessen, "Sub-micrometre accurate free-form optics by three-dimensional printing on single-mode fibres," Nat. Commun. 7, 11763 (2016).

${ }^{10}$ C. Crook, J. Bauer, A. G. Izard, C. Santos de Oliveira, J. Martins de Souza e Silva, J. B. Berger, and L. Valdevit, "Plate-nanolattices at the theoretical limit of stiffness and strength,” Nat. Commun. 11, 1579 (2020).

${ }^{11}$ Y. J. Liu, J. Y. Yang, Y. M. Nie, C. H. Lu, E. D. Huang, C. S. Shin, P. Baldeck, and C. L. Lin, "A simple and direct reading flow meter fabricated by two-photon polymerization for microfluidic channel," Microfluid. Nanofluid. 18, 427-431 (2015).

${ }^{12}$ R. Suriano, T. Zandrini, C. De Marco, R. Osellame, S. Turri, and F. Bragheri, "Nanomechanical probing of soft matter through hydrophobic AFM tips fabricated by two-photon polymerization," Nanotechnology 27, 155702 (2016).

${ }^{13}$ A. Marino, O. Tricinci, M. Battaglini, C. Filippeschi, V. Mattoli, E. Sinibaldi, and G. Ciofani, "A 3D real-scale, biomimetic, and biohybrid model of the blood-brain barrier fabricated through two-photon lithography," Small 14, 1702959 (2018).
${ }^{14}$ G. Vizsnyiczai, A. Búzás, B. L. Aekbote, T. Fekete, I. Grexa, P. Ormos, and L. Kelemen, "Multiview microscopy of single cells through microstructure-based indirect optical manipulation,” Biomed. Opt. Express 11, 945-962 (2020).

${ }^{15}$ F. Klein, T. Striebel, J. Fischer, Z. Jiang, C. M. Franz, G. von Freymann, M. Wegener, and M. Bastmeyer, "Elastic fully three-dimensional microstructure scaffolds for cell force measurements," Adv. Mater. 22, 868 (2010).

${ }^{16} \mathrm{C}$. N. LaFratta and T. Baldacchini, "Two-photon polymerization metrology: Characterization methods of mechanisms and microstructures," Micromachines 8, 101 (2017).

${ }^{17}$ Z. Bayindir, Y. Sun, M. J. Naughton, C. N. LaFratta, T. Baldacchini, J. T. Fourkas, J. Stewart, B. E. A. Saleh, and M. C. Teich, "Polymer microcantilevers fabricated via multiphoton absorption polymerization,” Appl. Phys. Lett. 86, 064105 (2005).

${ }^{18}$ H. B. Sun, K. Takada, and S. Kawata, "Elastic force analysis of functional polymer submicron oscillators," Appl. Phys. Lett. 79, 3173-3175 (2001).

${ }^{19}$ S. Ushiba, K. Masui, N. Taguchi, T. Hamano, S. Kawata, and S. Shoji, "Size dependent nanomechanics of coil spring shaped polymer nanowires," Sci. Rep. 5, 17152 (2015).

${ }^{20}$ I. S. Ladner, M. A. Cullinan, and S. K. Saha, "Tensile properties of polymer nanowires fabricated via two-photon lithography,” RSC Adv. 9, 28808-28813 (2019).

${ }^{21}$ S. Nakanishi, S. Shoji, S. Kawata, and H. Sun, "Giant elasticity of photopolymer nanowires,” Appl. Phys. Lett. 91, 063112 (2007).

22J. Bauer, A. G. Izard, Y. F. Zhang, T. Baldacchini, and L. Valdevit, "Programmable mechanical properties of two-photon polymerized materials: From nanowires to bulk,” Adv. Mater. Technol. 4, 1900146 (2019).

${ }^{23}$ T. Baldacchini, M. Zimmerley, C. H. Kuo, E. O. Potma, and R. Zadoyan, "Characterization of microstructures fabricated by two-photon polymerization using coherent anti-Stokes Raman scattering microscopy," J. Phys. Chem. B 113, 12663-12668 (2009).

${ }^{24}$ L. J. Jiang, Y. S. Zhou, W. Xiong, Y. Gao, X. Huang, L. Jiang, T. Baldacchini, J. F. Silvain, and Y. F. Lu, "Two-photon polymerization: Investigation of chemical and mechanical properties of resins using Raman microspectroscopy," Opt. Lett. 39, 3034-3037 (2014).

${ }^{25}$ K. Cicha, T. Koch, J. Torgersen, Z. Li, R. Liska, and J. Stampfl, “Young's modulus measurement of two-photon polymerized micro-cantilevers by using nanoindentation equipment," J. Appl. Phys. 112, 094906 (2012).

${ }^{26}$ K. Takada, D. Wu, Q. D. Chen, S. Shoji, H. Xia, S. Kawata, and H. B. Sun, "Size-dependent behaviors of femtosecond laser-prototyped polymer micronanowires," Opt. Lett. 34, 566-568 (2009).

${ }^{27}$ Z. Zhao, H. J. Qi, and D. N. Fang, "A finite deformation theory of desolvation and swelling in partially photo-cross-linked polymer networks for 3D/4D printing applications," Soft Matter 15, 1005-1016 (2019).

${ }^{28}$ C. S. Shin, T. J. Li, and C. L. Lin, "Alleviating distortion and improving the Young's modulus in two-photon polymerization fabrications," Micromachines 9, 615 (2018)

${ }^{29}$ S. J. Zhang, Y. Li, Y. K. Wang, L. P. Liu, H. D. Wang, Y. F. Xiao, H. Yang, and Q. Gong, "Controlling Young's modulus of polymerized structures fabricated by direct laser writing," Appl. Phys. A 118, 437-441 (2015).

${ }^{30}$ F. Klein, B. Richter, T. Striebel, C. M. Franz, G. von Freymann, M. Wegener, and M. Bastmeyer, "Two-component polymer scaffolds for controlled threedimensional cell culture," Adv. Mater. 23, 1341-1345 (2011).

${ }^{31} \mathrm{C}$. Schizas and D. Karalekas, "Mechanical characteristics of an Ormocomp (R) biocompatible hybrid photopolymer," J. Mech. Behav. Biomed. Mater. 4, 99-106 (2011).

${ }^{32}$ F. Qiu, L. Zhang, K. E. Peyer, M. Casarosa, A. Franco-Obregón, H. Choi, and B. J. Nelson, "Noncytotoxic artificial bacterial flagella fabricated from biocompatible ORMOCOMP and iron coating," J. Mater. Chem. B 2, 357-362 (2014).

${ }^{33}$ V. Telis, J. Telis-Romero, H. Mazzotti, and A. Gabas, "Viscosity of aqueous carbohydrate solutions at different temperatures and concentrations," Int. J. Food Prop. 10, 185-195 (2007)

${ }^{34} \mathrm{~K}$. Berg-Sorensen and H. Flyvbjerg, "Power spectrum analysis for optical tweezers," Rev. Sci. Instrum. 75, 594-612 (2004).

${ }^{35}$ G. Pesce, A. C. De Luca, G. Rusciano, P. A. Netti, S. Fusco, and A. Sasso, "Microrheology of complex fluids using optical tweezers: A comparison with macrorheological measurements,” J. Opt. A 11, 034016 (2009).

${ }^{36}$ E. Schaffer, S. Norrelykke, and J. Howard, "Surface forces and drag coefficients of microspheres near a plane surface measured with optical tweezers," Langmuir 23, 3654-3665 (2007). 